\title{
Cortical regions associated with different aspects of object recognition performance
}

\author{
JANE E. JOSEPH and ALISON B. FARLEY \\ University of Kentucky Medical Center, Lexington, Kentucky
}

\begin{abstract}
In the present object recognition study, we examined the relationship between brain activation and four behavioral measures: error rate, reaction time, observer sensitivity, and response bias. Subjects perceptually matched object pairs in which structural similarity (SS), an index of structural differentiation, and exposure duration (DUR), an index of task difficulty, were manipulated. The SS manipulation affected the fMRI signal in the left anterior fusiform and parietal cortices, which in turn reflected a bias to respond same. Conversely, an SS-modulated fMRI signal in the right middle frontal gyrus reflected a bias to respond different. The DUR manipulation affected the fMRI signal in occipital and posterior fusiform regions, which in turn reflected greater sensitivity, longer reaction times, and greater accuracy. These findings demonstrate that the regions most strongly implicated in processing object shape (SS-modulated regions) are associated with response bias, whereas regions that are not directly involved in shape processing are associated with successful recognition performance.
\end{abstract}

The primate ventral processing stream (VPS) is critically involved in object recognition, as has been evidenced by a number of animal lesion and electrophysiological studies (Tanaka, 1996; Ungerleider \& Mishkin, 1982), as well as human lesion (Bauer, 1993; Farah, 1990) and functional neuroimaging (Malach et al., 1995; Schacter et al., 1995) studies. However, a persistently debated topic is the functional organization of the VPS for the recognition of different categories of objects (Martin \& Caramazza, 2003). Building on findings from human lesion studies (e.g., Hillis \& Caramazza, 1991; Warrington \& Shallice, 1984), functional neuroimaging studies have revealed dissociated patterns of brain activation, so that different regions of the VPS tend to respond preferentially to animals, manufactured objects, human faces, body parts, and places (H. Damasio, Grabowski, Tranel, Hichwa, \& A. R. Damasio, 1996; Downing, Jiang, Shulman, \& Kanwisher, 2001; Epstein \& Kanwisher, 1998; Kanwisher, McDermott, \& Chun, 1997; Martin, Wiggs, Ungerleider, \& Haxby, 1996; Okada et al., 2000; Spitzer et al., 1998), suggesting cortical specialization for the recognition of different object categories. However, other accounts have proposed that the representation of different object categories is more distributed than localized (Haxby,

This work was previously presented in poster form at the 11th Annual Meeting of the Cognitive Neuroscience Society, New York (2004). This research was supported in part by grants from the National Science Foundation (BCS-0224240) and the National Institute of Mental Health (R01 MH063817). We thank Gerry Piper, Robin Avison, and Agnes Bognar for their assistance with data collection and Nick Steinmetz for his technical assistance. We also thank the subjects who volunteered for the study. Correspondence concerning this article should be addressed to J. E. Joseph, Department of Anatomy and Neurobiology, University of Kentucky Medical Center, 800 Rose Street, Davis-Mills Building, Room 308, Lexington, KY 40536 (e-mail: jjoseph@uky.edu).
Ishai, Chao, Ungerleider, \& Martin, 2000; Joseph \& Gathers, 2002; Price, Noppeney, Phillips, \& Devlin, 2003; Tyler \& Moss, 2001). In either case, the principle driving the functional organization of the VPS has yet to be isolated.

In the present study, functional magnetic resonance imaging (fMRI) was used to explore a potential explanatory principle for category specificity in brain activation patterns in the VPS - namely, structural similarity (SS). Representations of an object's three-dimensional (3-D) structure, called structural descriptions, play a pivotal role in object recognition (Biederman, 1987; Marr \& Nishihara, 1978). The degree of overlap in 3-D structure among objects in the same category determines the efficiency of recognition (A. R. Damasio, H. Damasio, \& Van Hoesen, 1982; Humphreys, Riddoch, \& Quinlan, 1988). For example, natural objects take longer to name than do manufactured objects (Humphreys et al., 1988), presumably because natural objects, in general, overlap more in terms of structural descriptions than do manufactured objects. In addition, shape or structural information may be more important for the recognition of natural objects than for manufactured object categories (Warrington \& Shallice, 1984). During the process of naming or identifying an object, competing structural representations for objects are activated, requiring the observer to distinguish the target from competing representations. One way to resolve this competition is to engage additional perceptual or structural analyses, which we refer to as structural differentiation in the present article (see also Price et al., 2003). Consequently, objects that are very similar at the level of structural descriptions will be more difficult to discriminate from each other than objects that are not structurally similar. We suggest not only that the process of structural differentiation underlies different kinds of object recognition tasks (naming, identi- 
fication, matching, and episodic recognition), but also that this process is central in many competing theories of object recognition.

Specifically, many authors have now proposed that different demands on perceptual or structural differentiation contribute to taxonomic category distinctions. These proposals can be found in the cognitive processing (Bruce \& Humphreys, 1994), cognitive neuropsychology (A. R. Damasio et al., 1982), and functional neuroimaging (Gauthier, Tarr, Anderson, Skudlarski, \& Gore, 1999; Joseph \& Gathers, 2003; Joseph, Jones, Zeffiro, \& Eden, 2000; Price et al., 2003; Tyler et al., 2003; Tyler et al., 2004) literature. For example, faces represent the extreme end of the continuum of structural similarity (Bruce \& Humphreys, 1994) and neural substrates for face recognition are activated by a contrived set of stimuli that also make high demands on perceptual differentiation (Gauthier et al., 1999). In some of these studies, however, differences in perceptual differentiation are often inferred, rather than objectively or normatively determined. For example, natural objects are presumed to require more structural differentiation than do manufactured objects, and subordinate- or basic-level categorization and naming are also expected to make greater demands on structural differentiation. Yet, in many cases, structural differentiation is not operationalized. Exceptions are studies in which structural similarity is operationalized by degree of contour overlap of object drawings normalized to the same orientation (Humphreys et al., 1988; Tranel, Logan, Frank, \& Damasio, 1997), ratings of number of shared components among objects of a given category (Humphreys et al., 1988 ), or judgments of how visually similar an object is relative to other members of its category, referred to as homomorphy (Tranel et al., 1997). Operational definitions such as these directly address the visual similarity or dissimilarity of objects within different taxonomic categories.

In our own work, we have explored demands on structural differentiation, using normative SS ratings (Joseph, 1997; Joseph \& Proffitt, 1996) that reflect varying degrees of structural overlap between two objects. In one fMRI study (Joseph et al., 2000), subjects named line drawings of animals in which the pairwise similarity among the animals was either high or low. Naming animals in the high-SS condition was more difficult than naming animals in the low-SS condition and activated more anterior aspects of the inferior temporal and fusiform gyri, which are critical components of the VPS. Moreover, the low-SS naming condition activated only posterior aspects of the VPS. ${ }^{1}$ We argued that greater demands on differentiating high-SS animals at the level of structural descriptions resulted in activation further downstream in anterior portions of the VPS. This proposal for an anterior shift in fusiform activation as demands on structural or perceptual differentiation increase has also been discussed by other authors (Gerlach, Law, Gade, \& Paulson, 1999; Price et al., 2003). Low-SS animals, in contrast, can be differentiated at the perceptual or structural level of processing, which, we argued, makes greater demands on posterior VPS regions. A similar finding has been reported in Tyler et al. (2004), in which basic-level naming activated medial temporal cortices, whereas activation associated with domain-level naming did not extend into the same anterior aspects of the VPS activated by basic-level naming. Although it is likely a safe assumption that basic-level naming makes greater demands on structural differentiation than does domain-level naming, demands on structural differentiation are not directly addressed with a categorization-level manipulation, whereas the manipulation of structural similarity is a more direct index of degree of structural differentiation.

In another fMRI study (Joseph \& Gathers, 2003), subjects decided whether pairs of animals that varied across four SS levels were the same or different. As was expected, the subjects took longer to respond and made more errors with the high-SS different pairs than with low-SS different pairs. This greater difficulty was driven by the greater demands on structural differentiation in the high-SS conditions. In addition, similar to the previous naming study, high-SS matching produced a greater fMRI signal in anterior aspects of the fusiform gyrus than did low-SS matching, and low-SS matching produced a greater fMRI signal in posterior aspects of the VPS. In that same matching study, we also examined 3-D shapes that were not easily named but that varied across three SS levels. Again, high-SS shapes activated anterior aspects of the fusiform gyrus, whereas low-SS shapes activated posterior VPS regions (the primary visual cortex). Taken together, these findings suggest that greater demands on structural differentiation, as indexed by higher SS, recruit more anterior aspects of the VPS. This anterior VPS activation is not necessarily linked to semantic processing, given that meaningless 3-D shapes also activated similar anterior VPS regions. Hence, the first goal of the present study was to further test the viability of structural similarity as a potential explanatory principle for the functional organization of the VPS. We predicted that high-SS matching engages more anterior aspects of the VPS than does low-SS matching and that low-SS matching preferentially engages more posterior VPS regions than does high-SS matching, as in our previous studies (Joseph \& Gathers, 2003; Joseph et al., 2000).

An alternative explanation is that the anterior VPS activation does not reflect greater demands on structural differentiation but, instead, reflects generalized task difficulty, given that the high-SS conditions induced longer reaction times (RTs) and higher error rates (ERRs). Although we had previously addressed these concerns (Joseph \& Gathers, 2003), in the present study we examined the relationship between fMRI signal and task performance in more depth, using a similar matching task in which we parametrically varied SS levels. To elucidate the relationship between task performance and fMRI signal, we examined four measures of performance: RT, ERR, bias, and sensitivity (SENS), with the latter two measures defined within a signal detection framework 
(Donaldson, 1992; MacMillan \& Creelman, 1991). By using several measures of performance, we could more fully characterize object recognition performance and more carefully interpret cognitive processing associated with brain activation patterns. Reliance on a single performance measure to infer cognitive processing is often ambiguous. For example, if an observer takes longer to respond in experimental Condition $\mathrm{A}$ than in $\mathrm{B}$, one might conclude that Condition A is more difficult than Condition B. However, ERRs must also be examined in this case, in order to rule out a speed-accuracy tradeoff. If ERRs are the same across both conditions or if ERRs increase as RT increases, the conclusion that Condition A is more difficult than Condition B is valid. However, the longer RTs in Condition A may be accompanied by low ERRs, and the shorter RTs in Condition B may be accompanied by high ERRs. This type of speed-accuracy tradeoff calls into question the interpretation that one task is more difficult than another, because the tradeoff simply reflects a strategy in which fast responding at the expense of more errors is adopted in one condition and the opposite strategy is adopted in another condition. Without knowing how both RT and ERR change, interpretation about task difficulty is ambiguous.

Due to the possibility of speed-accuracy tradeoffs, we suggest it is also helpful to examine observer SENS and bias measures to add to the interpretation of behavior. SENS refers to the ability of the observer to discriminate targets from noise, whereas bias refers to the strategy adopted by an observer in order to maximize hits (i.e., correct detection of a target) or minimize false alarms (i.e., incorrectly identifying noise as a target). In theory, SENS and bias are independent constructs, so that two equally sensitive observers may exhibit different response biases (MacMillan \& Creelman, 1991). A liberal bias reflects a criterion setting in which most signals are detected, but at the expense of many false alarms, whereas a conservative bias reflects a stricter criterion setting in which some signals are rejected as noise. Note that measures of response bias do not necessarily reflect task difficulty, as do RT, ERR, and to some extent, SENS. Consequently, to show that activation in a brain region correlates with response bias would not likely indicate generalized task difficulty.

The need to correlate brain activation with different performance measures has now been emphasized by several authors (e.g., Garrett et al., 2000; Gur et al., 1997), and object and face recognition studies are increasingly incorporating measures of performance in the interpretation of brain activation patterns (Bar et al., 2001; GrillSpector, Kourtzi, \& Kanwisher, 2001; James, Humphrey, Gati, Menon, \& Goodale, 2000). For example, in the study by Bar et al., briefly presented and masked pictures of objects were difficult to recognize upon initial presentation. With repeated presentations of the pictures, however, subjects were able to recognize the objects successfully. Their results showed that fMRI signal in the anterior fusiform gyrus was associated with successful recognition. Specifically, the fMRI signal increased linearly as subjective ratings of recognition success increased. More posterior fusiform regions and early visual areas did not show a response that was driven by subjective ratings of successful recognition. The latter finding of systematic changes in brain activation as a function of task performance strengthens the conclusion that anterior fusiform regions are specifically involved in object recognition.

Although correlations between the magnitude of brain activation and performance measures have now been examined in many functional neuroimaging studies, to our knowledge, no functional neuroimaging studies of object recognition have been concerned with how both sensitivity and bias, as measured in a signal detection framework, correlate with brain activation signal. Consequently, our second goal was to correlate SENS, bias, RT, and ERR with fMRI signal in order to better characterize which aspects of performance drive brain activation patterns in an object recognition task. More specifically, are the anterior fusiform regions previously implicated in structural differentiation more strongly correlated with processing speed, accuracy in responding, observer SENS, or shifts in criterion settings? Each of these dependent measures reflects a somewhat different aspect of cognitive processing, but few functional neuroimaging studies of object recognition have addressed this.

Another approach to understanding how task difficulty and task-relevant performance relate to fMRI signal and structural processing is to manipulate task difficulty independently from the demands on structural processing. In a previous unpublished behavioral study within our lab, 15 subjects performed an animal-matching task (similar to that used in Joseph \& Gathers, 2003) in which four levels of SS and three levels of exposure duration (DUR, 200,400 or $1900 \mathrm{msec}$ ) were factorially combined. RTs and ERRs increased as SS level increased, which was expected given that high-SS conditions made greater demands on structural differentiation. These demands were manifest in terms of both RT and ERR, with no evidence for a speed-accuracy tradeoff. In terms of DUR, however, RTs were shorter for the shorter DURs but ERRs were higher, suggesting a speed-accuracy tradeoff for the DUR manipulation. Importantly, DUR did not alter the behavioral RT and the error patterns driven by SS (i.e., the interaction was not significant). This behavioral study showed that DUR did not alter the RT or error functions associated with SS, but it did affect overall reaction time and accuracy. In other words, the DUR manipulation influenced task difficulty independently from the SS manipulation. With respect to RT, longer durations were more difficult (if a longer RT is taken as an index of greater difficulty), but with respect to errors, longer durations were less difficult. Consequently, the third goal of the present fMRI study was to vary DUR in an attempt to manipulate task difficulty apart from the demands on structural processing. We predicted that anterior fusiform regions that appear to be driven by greater demands on structural dif- 
ferentiation would not also be driven by generalized task difficulty, as indexed by poorer performance as a function of DUR.

We employed a block design in which the two variables of interest were factorially combined and manipu- lated across blocks (see Figure 1). To isolate patterns of brain activation associated with the independent variables of interest and performance measures, we used cross-correlation analysis with typical reference waveforms for each of the cross-correlations performed illus-

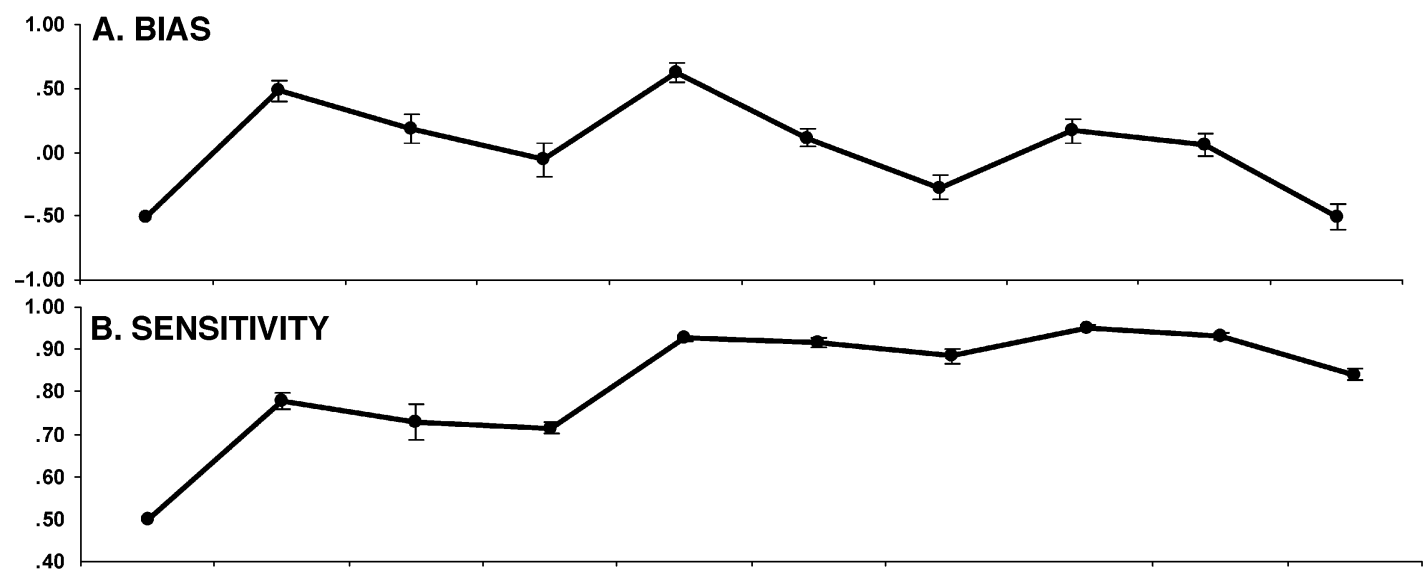

C. ERR

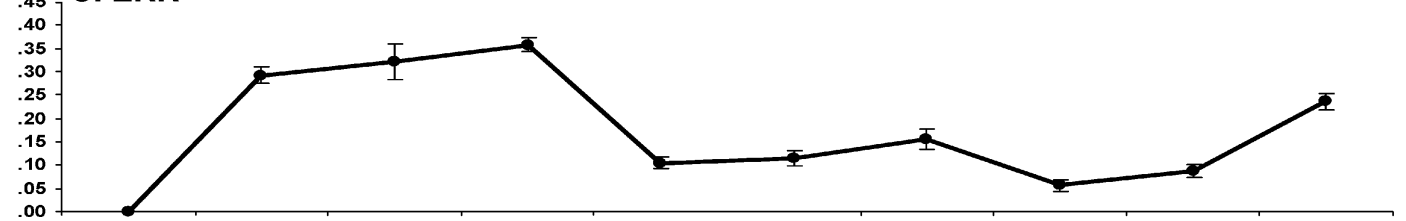

D. RT
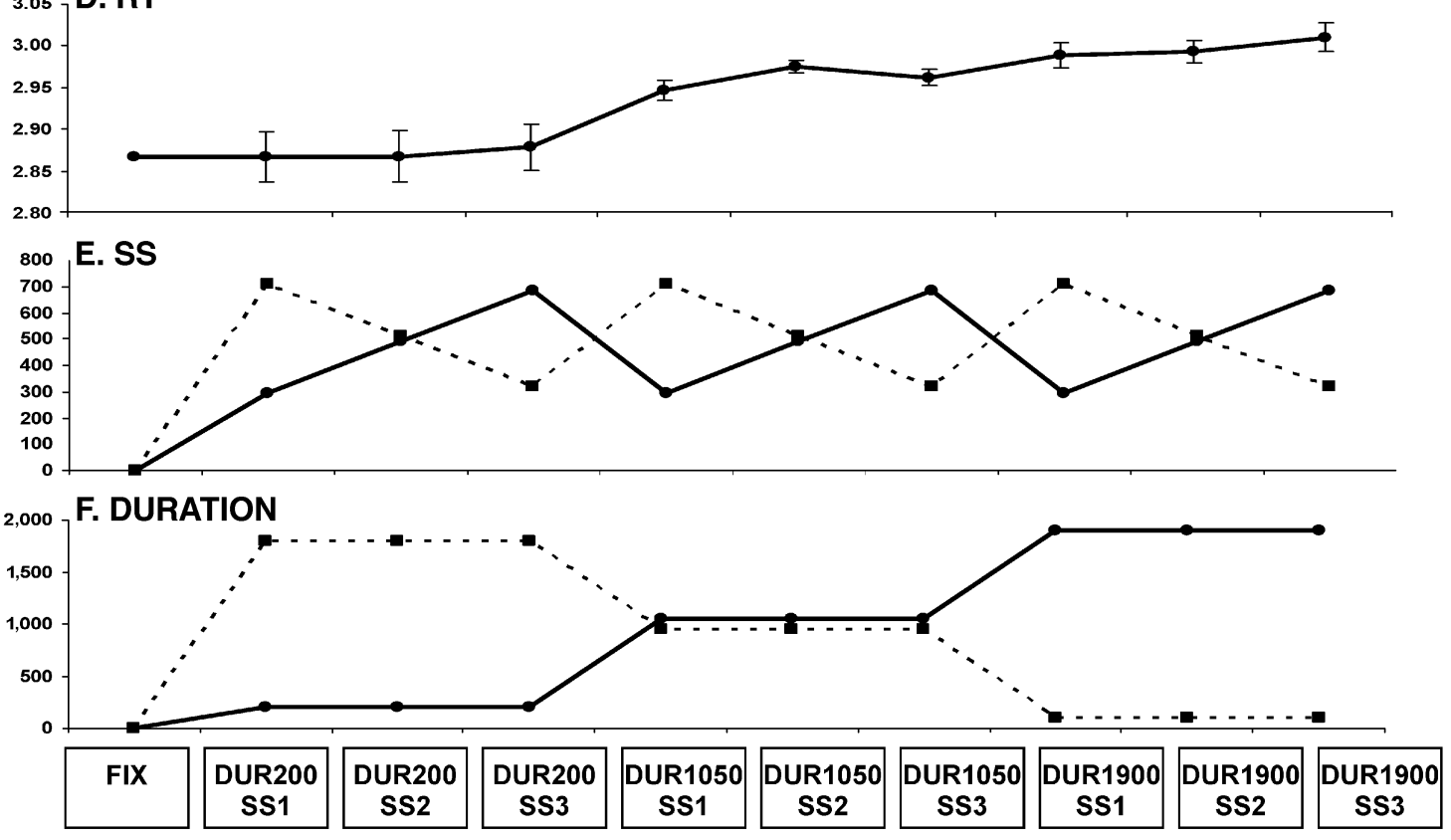

EXPERIMENTAL CONDITION

Figure 1. Sample reference waveforms used for cross-correlations in the present study. The $x$-axis represents the experimental design in which a visual fixation condition was used as a baseline, and nine experimental conditions reflect the factorial combination of the exposure duration (DUR) and structural similarity (SS) variables. For panels A through $D$, the $y$-axis reflects the group-averaged values for each of the four performance measures: reaction time (RT), error rate (ERR), sensitivity (SENS), and bias. For panels $E$ and $F$, the $y$-axis represents parametrically varied values of the two independent variables DUR and SS (solid lines) or the complements of these two waveforms (dotted lines). Error bars reflect within-subjects confidence intervals (Loftus \& Masson, 1994). 
trated in Figure 1. RT refers to the average time to respond same or different within a block of trials. ERR refers to the percentage of incorrect responses over a block of trials. Two measures from signal detection theory (Donaldson, 1992) were also examined. SENS refers to the ability of the observer to discriminate targets (same trials) from distractors (different trials) in a block of trials; bias refers to a tendency to respond same (liberal criterion setting) versus different (conservative criterion setting) within a block of trials. The three main objectives were (1) to replicate previous findings (Joseph \& Gathers, 2003; Joseph et al., 2000) that the VPS is involved in structural differentiation during object recognition, as tapped by the SS manipulation, (2) to further characterize the type of cognitive and perceptual processing subserved by the VPS by correlating fMRI signal with a variety of different performance measures, and (3) to manipulate task difficulty apart from the demands on structural object processing, to rule out the interpretation that VPS activation reflects generalized task difficulty, rather than structural object processing.

\section{METHOD}

\section{Subjects}

Twelve paid adult volunteers were scanned ( 6 females and 6 males, mean age of 24 years, all right-handed, with normal or corrected-to-normal vision). No subject had a history of medical, neurological, or psychiatric illness; however, 1 subject received a blunt force to the head, after being enrolled in the study, 2 days prior to scanning but had no lingering effects of this injury. The subjects gave written consent, in accord with the guidelines of the University of Kentucky Institutional Review Board.

\section{Stimuli and Procedure}

Line drawings of animals from several different classes (birds, mammals, reptiles, amphibians, fish, and insects) served as stimuli.
In a previous rating study (Joseph, 1997), 22 subjects viewed 195 pairs of natural objects two times and rated the similarity of the two items of a pair in terms of 3-D volumetric structure. In that rating study, the subjects were instructed to focus on the 3-D structure and to ignore similarity of the object outlines, sizes, orientations, and taxonomic categories. Using a mouse, the subjects moved a marker along the analogue rating scale, which was anchored by the labels least similar (corresponding to a value of 0 ) and most similar (corresponding to a value of 1,000). The distribution of structural similarity ratings from that previous study was divided into three equal groups to determine the assignment of animal pairs to each of the three SS levels in the present study (20 pairs per SS level; see examples in Figure 2).

The subjects practiced the present matching task before entering the MRI scanner by viewing the same object pairs for as long as they needed to learn the correct matches, followed by a practice session of 12 same and 12 different trials in random order (the majority of the subjects needed only one practice session). On each trial of the fMRI study, one picture appeared above and one below fixation (the entire display subtended an approximate visual angle of $4^{\circ}$ ). Each pair appeared for 200, 1,050, or 1,900 msec depending on the experimental condition (Figure 1), followed by $1,800,950$ or $100 \mathrm{msec}$ of fixation, respectively. The subjects decided whether the two pictures represented the same or a different object and pressed one of two buttons on an MRI-compatible response box, using index (same) and middle (different) fingers. The subjects were instructed to fixate the crosshair at all times and to make responses as quickly and as accurately as possible (the subjects could respond at any point during the $2-\mathrm{sec}$ trial). No feedback was given during the experiment.

\section{fMRI Design and Data Acquisition}

All the subjects completed 3 functional runs, for a total of 36 runs in this study, but 8 runs were omitted due to excessive head motion (each subject had at least 1 acceptable run). Within each run, nine experimental task epochs ( 3 DUR levels $\times 3$ SS levels) in pseudorandom order alternated with eight fixation epochs. Eight volumes were collected per task epoch, and two volumes per fixation epoch. Twenty-four experimental trials occurred in each task epoch, with same and different trials occurring with equal frequency. Although

\begin{tabular}{|l|c|c|c|}
\hline $\begin{array}{c}\text { Structural } \\
\text { Similarity } \\
\text { Level }\end{array}$ & Same trials & Different trials & $\begin{array}{c}\text { Mean SS } \\
\text { rating (SD) }\end{array}$ \\
\hline SS1 & SS2 & & \\
SS3 & &
\end{tabular}

Figure 2. Sample object pairs for each of the three levels of structural similarity (SS1-SS3), with mean SS ratings (with standard deviations) for different trials shown in the last column (Joseph, 1997). 
each task epoch contained different pairs from only one SS level, the same pairs were randomly determined within each epoch, so that there was no systematic variation of same pairs across blocks and, hence, across SS levels. A Siemens Vision 1.5 Tesla magnet equipped with a head coil for whole-brain imaging and a T2*weighted gradient echo sequence were used to collect functional brain images (40-msec echo time, 6 -sec repetition time, $64 \times 64$ matrix, 230-mm field of view, $463-\mathrm{mm}$ slices with a $20 \%$ gap acquired in ascending order). The relatively longer repetition time used in the present study ( $6 \mathrm{sec}$ ) enabled us to collect high-resolution images (3.6-mm cubic voxels) with whole-brain coverage. In addition, we wanted to keep the imaging parameters and design as similar as possible to those in our previous study (Joseph \& Gathers, 2003), in order to replicate the effect of SS, which meant having a longer repetition time and a block design.

\section{Data analysis}

MEDx software (Sensor Systems, Sterling, VA) was used for fMRI data analysis, and SPSS (SPSS, Inc., Chicago) was used for all scalar statistical analyses. After discarding the first two fixation volumes of each subject's time series, the remaining echo-planar images were realigned to the mean intensity image of the time series, using a six-parameter rigid body model and the 3 -D scanline chirp- $Z$ algorithm with a least-squares cost function for resampling (Woods, Cherry, \& Mazziotta, 1992). Gaussian filtering (6- $\mathrm{mm}^{3}$ filter and kernel size of 9 pixels), intensity normalization, and high-pass filtering (period of $120 \mathrm{sec}$ ) were applied to the data. For each subject and each run, nine mean intensity images for the various experimental conditions (DUR [3] $\times$ SS [3]) were spatially normalized using the standardized stereotactic system of Talairach and Tournoux (1988).

After data preprocessing, we performed eight separate crosscorrelations for each subject and each run, using the reference waveforms as depicted in Figure 1. For the DUR and SS correlations, the same reference waveform was used for all the subjects, given that these waveforms captured the parametrically varied values of each experimental variable of interest. Two reference waveforms were constructed for DUR, and two for SS. ${ }^{2}$ The solid waveforms in Figure 1 represent the averaged manipulated values for each variable. The dotted waveforms are the complements of the actual data values, with the exception of the value assigned to the fixation (FIX) condition. The purpose of having two waveforms for each independent variable is to detect brain regions sensitive to higher levels of each variable, as well as to detect brain regions sensitive to lower levels of the variables. Note that simply using the negative correlation map of the cross-correlation based on the actual values would reflect activation due to the baseline (or deactivation). In the present approach, both waveforms should detect activation greater than that in the baseline condition (FIX), but higher values of the manipulated variable are more strongly weighted with the positive (solid) waveforms, and lower values of the variable are more strongly weighted with the complement (dotted) waveforms.

The waveforms for the four performance measures (RT, ERR, bias, and SENS) were constructed on the basis of each individual subject's data. The FIX condition was assigned the minimum value in the case of RT and bias, the value 0 in the case of ERR, and the chance performance value (.5) in the case of SENS. All of the crosscorrelations yielded statistical maps of Pearson $r$ values, converted into $z$ scores. The resulting $z$ maps were then averaged together in a fixed effects approach (Lazar, Luna, Sweeney, \& Eddy, 2002; Papoulis, 1965) 3 to yield a group statistical map for each measure depicted in Figure 1. Cluster detection (Friston, Worsley, Frackowiak, Mazziotta, \& Evans, 1994) was then applied to the group $z$ maps $(p<.001$, Bonferroni corrected) to yield regions of interest (ROIs) for the additional analyses described below. No clusters sensitive to ERR or the complement of the DUR reference waveform survived the cutoff.

Within each of the 40 ROIs that emerged from these crosscorrelations, we determined the percent signal change relative to FIX for each of the nine time points that reflected the combination of SS and DUR levels (Figure 1). We then performed two types of analyses within each cluster. In one analysis, we conducted randomeffects repeated measures analyses of variance (ANOVAs) that examined the effects of SS $(1,2$, or 3$)$, DUR $(200,1,050$, or 1,900) and the SS $\times$ DUR interaction on percent signal change. In another analysis, we conducted scalar correlations between percent signal change and the reference waveforms depicted in Figure 1. In these scalar correlations, the SS and SS-complement correlations are negatives of each other, as are the DUR and DUR-complement correlations; therefore, only the results from the SS and DUR correlations are included, because the correlations with their complements

Table 1

Brain Regions Showing Significant Correlations Between fMRI Signal and Performance and Significant Effects of Structural Similarity (SS) and Exposure Duration (DUR)

\begin{tabular}{|c|c|c|c|c|c|c|c|c|c|c|c|c|}
\hline \multirow[b]{2}{*}{ Region } & \multicolumn{9}{|c|}{ Correlations (Pearson $r$ ) } & \multicolumn{3}{|c|}{ ANOVAs ( $F$ Values) } \\
\hline & $\mathrm{BA}$ & Size & $x, y, z$ & SS & DUR & RT & ERR & SENS & BIAS & SS & DUR & $\mathrm{SS} \times \mathrm{DUR}$ \\
\hline \multicolumn{13}{|c|}{ Occipital Areas } \\
\hline L middle occipital (gyrus) & 18 & 44 & $-30,-93,+1$ & -.02 & $.81 *$ & $.81^{*}$ & $-.69^{*}$ & $.65 \dagger$ & -.36 & n.s. & $5.1 *$ & n.s. \\
\hline $\mathrm{L}$ inferior occipital $\mathrm{g}$. & 18 & 276 & $-39,-86,-6$ & .09 & & .9 & $-.74 *$ & $.71 *$ & -.42 & n.s. & $11.3 *$ & \\
\hline $\mathrm{L}$ inferior occipital $\mathrm{g}$. & 18 & 1,546 & $-37,-80,-9$ & .16 & .8 & $.89 *$ & $-.69 *$ & $.66 \dagger$ & -.48 & n.s. & $18.2 *$ & $5.3^{*}$ \\
\hline $\mathrm{L}$ inferior occipital $\mathrm{g}$. & 18 & 1,079 & $-41,-79,-8$ & .18 & & .8 & $-.64 \dagger$ & $.62 \dagger$ & -.53 & n.s. & $13.8^{*}$ & $5.9 *$ \\
\hline $\mathrm{R}$ lingual g. & 18 & 5 & $+22,-78,-9$ & -.37 & & .6 & $-.75^{*}$ & $.72 *$ & .10 & $5.4^{*}$ & $8.7^{*}$ & $3.9 *$ \\
\hline dle occipital g. & 18 & 1,809 & $+36,-75,-10$ & .03 & & .8 & $-.67 *$ & $.65 \dagger$ & -.40 & n.s. & $26.4 *$ & $8.8^{*}$ \\
\hline R superior occipital g. & 19 & 9 & $+30,-74,+27$ & -.07 & $.75^{*}$ & $.80^{*}$ & $-.69 *$ & $.66 \dagger$ & -.21 & n.s. & $4.2 *$ & n.s. \\
\hline \multicolumn{13}{|c|}{ Temporal, Frontal, Parietal, Subcortical Areas } \\
\hline $\mathrm{R}$ posterior $\mathrm{f}$ & 19 & 1,518 & $+37,-73,-11$ & .07 & $.86^{*}$ & $.87 *$ & $-.66 \dagger$ & $.64 \dagger$ & -.43 & n.s. & $20.8 *$ & $6.5^{*}$ \\
\hline $\mathrm{R}$ mid fusiform $\mathrm{g}$. & 19 & 799 & $+40,-67,-11$ & -.10 & $.83 *$ & $.84^{*}$ & $-.70^{*}$ & $.68^{*}$ & -.27 & n.s. & $13.2 *$ & $5.5^{*}$ \\
\hline $\mathrm{L}$ anterior fusiform $\mathrm{g}$. & 37 & 7 & $-41,-47,-21$ & .41 & $.82 *$ & $.79 *$ & -.50 & .46 & $-.76^{*}$ & n.s. & $7.5^{*}$ & n.s. \\
\hline $\mathrm{L}$ anterior fusiform $\mathrm{g}$. & 37 & 16 & $-43,-46,-25$ & $.61 \dagger$ & $.61 \dagger$ & .59 & -.28 & .22 & $-.87^{*}$ & $5.3^{*}$ & $4.1^{*}$ & n.s. \\
\hline $\mathrm{L}$ precuneus & 7 & 102 & $-29,-63,+37$ & $.73^{*}$ & .45 & .34 & .03 & -.07 & $-.85^{*}$ & $6.7 *$ & n.s. & n.s. \\
\hline $\mathrm{R}$ middle frontal $\mathrm{g}$. & 6 & 30 & $+27,+1,+69$ & $-.88 *$ & -.33 & -.33 & -.08 & .10 & $.91 *$ & $8.9^{*}$ & n.s. & n.s. \\
\hline $\mathrm{R}$ cerebellum & & 13 & $+16,-55,-21$ & $.76^{*}$ & -.45 & -.37 & $.65 \dagger$ & $-.66 \dagger$ & $-.60 \dagger$ & $3.4 \dagger$ & n.s. & n.s. \\
\hline
\end{tabular}

Note-Cluster size is expressed in voxels after transformation into Talairach space (Talairach \& Tournoux, 1988), in which each voxel is $8 \mathrm{~mm}^{3}$. BA, Brodmann's area; ERR, error rate; L, left; n.s., not significant; R, right; RT, reaction time; SENS, sensitivity; $x$, medial-lateral coordinate; $y$, anterior-posterior coordinate; $z$, inferior-superior coordinate. ${ }^{*} p<.05 . \quad{ }^{\dagger} p<.086$. 

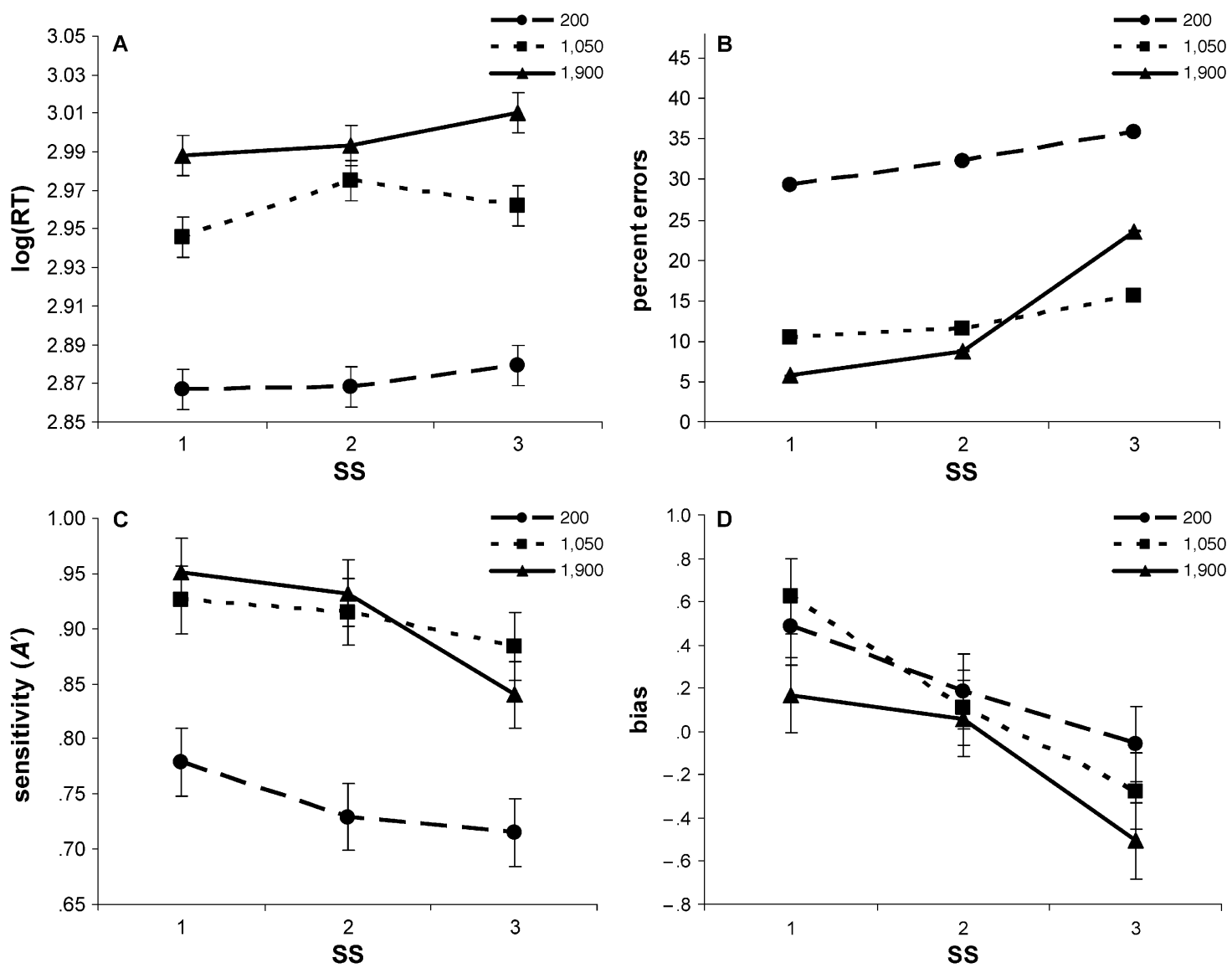

Figure 3. Main effects of structural similarity (SS) and exposure duration (DUR) and the SS $\times$ DUR interaction for each of the four dependent measures: (A) reaction time (RT), (B) error rate, (C) sensitivity, and (D) bias. Error bars reflect within-subjects confidence intervals (Loftus \& Masson, 1994).

are redundant. ${ }^{4}$ The scalar correlations enabled us to relate fMRI signal to performance measures, whereas the ANOVAs allowed us to examine the effect of the manipulated variables on fMRI signal change. Because the ANOVAs are random-effects analyses, they are inferential in nature and account for both within- and betweensubjects variability. Moreover, they are critical for determining whether the effects we observed were consistent across subjects within our sample. Table 1 lists those regions in which fMRI signal showed both a main effect of either SS or DUR according to the ANOVAs and a significant or marginally significant correlation with any of the two independent or four dependent variables we examined.

\section{RESULTS}

\section{Behavioral Results}

Log-transformed RTs from individual trials greater than three standard deviations from the mean were considered outliers $(0.5 \%$ of the data). Only correct logtransformed RTs were submitted to analyses ( $79 \%$ of the data). Due to constraints on the length of fMRI studies, the number of trials per cell of the design was not sufficient to use a parametric approach to signal detection
(MacMillan \& Creelman, 1991); hence, we used a nonparametric approach to compute bias and SENS (Donaldson, 1992). Bias values could range from -1 to 1 , with negative values indicating a bias to respond same, or a liberal bias. Higher SENS values indicate greater discrimination between same and different pairs, and a SENS value of .5 indicates chance performance. Figure 3 shows the group-averaged RT, ERR, bias, and SENS values as a function of the SS and DUR variables (collapsed across same and different trials for RT and ERR). A two-way repeated measures ANOVA using the multivariate approach (O’Brien \& Kaiser, 1985) was conducted for each performance measure separately with SS $(1,2$, or 3$)$ and DUR $(200,1,050$, or $1,900 \mathrm{msec})$ manipulated within subjects. Although two of the performance measures were significantly negatively correlated $(r=-.97, p<$ .0001 , for SENS and ERR), the other intercorrelations were not significant $(p>.3)$; therefore, we did not use a multivariate approach for the four performance measures. In addition, given that we used the multivariate approach to repeated measures, we did not have enough degrees of freedom for a full multivariate approach; there- 
fore, we analyzed the performance measures in four separate ANOVAs.

The ANOVAs showed that all four dependent measures were affected by SS level (Figure 3). At higher SS levels, the subjects took longer to respond $[F(2,22)=$ $8.8, p<.01]$, made more errors $[F(2,22)=29.5, p<$ $.0001]$, adopted a more liberal criterion setting [i.e., bias to respond same; $F(2,22)=52.8, p<.0001]$, and were less sensitive to differences between same and different pairs $[F(2,10)=49.2, p<.0001]$. DUR also had a significant effect on all the dependent measures. The subjects responded more quickly at shorter durations than at longer durations $[F(2,10)=11.7, p<.01]$ but made more errors $[F(2,22)=110.8, p<.0001]$, indicating a speed-accuracy tradeoff. At longer durations, the subjects were biased to respond same $[F(2,10)=15.3, p<$
$.01]$, but they were better able to discriminate matches from nonmatches $[F(2,10)=36.4, p<.0001]$. Although both independent variables significantly influenced performance, the effect of DUR was greater in magnitude than the effect of SS on all the dependent measures except bias.

Finally, the two-way interaction between SS and DUR was significant for all four dependent measures, indicating that the two factors were not completely independent $[F(4,8)=8.9, p<.01$, for RT; $F(4,8)=21.4, p<.0001$, for ERR; $F(4,8)=7.5, p<.01$, for SENS; $F(4,44)=$ $4.8, p<.01$, for bias], unlike in our previous unpublished study conducted outside of the MRI scanner. As is shown in Figure 3, the trends reflected by the main effect of SS (e.g., increased RT as SS increased, or decreased SENS as SS increased) were preserved at all the expo-

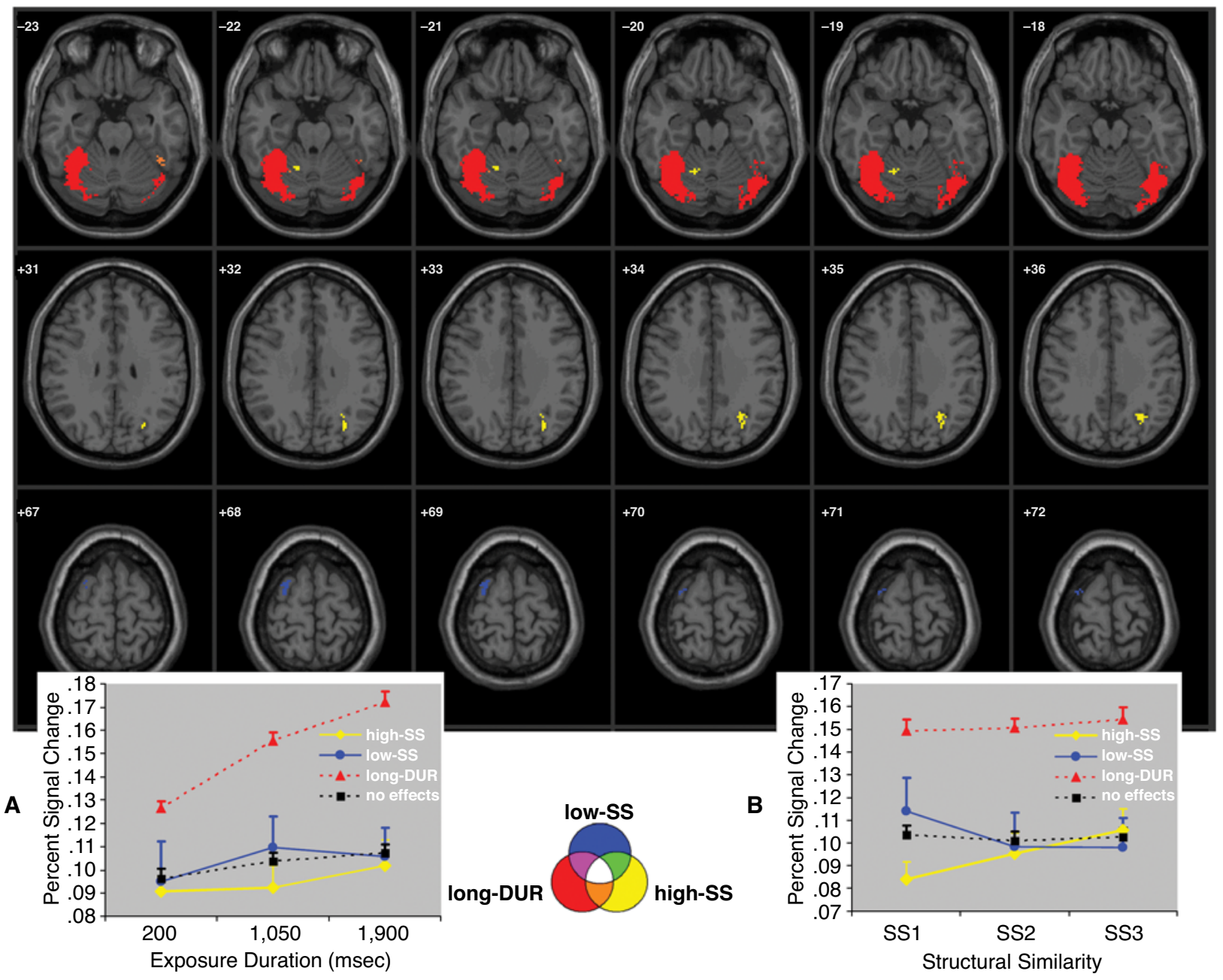

Figure 4. Brain activation in 18 selected axial slices throughout the cortex. The upper left-hand value in each slice represents the inferior-superior dimension of Talairach space (Talairach \& Tournoux, 1988). Red voxels represent brain regions that were sensitive to longer durations, yellow voxels represent brain regions that were sensitive to high structural similarity (SS) levels, and blue voxels represent brain regions that were sensitive to low SS levels. Orange voxels represent regions that were sensitive to both longer durations and high SS. The statistical threshold for all activations was $p<.001$, corrected. Inset A: percent signal change as a function of exposure duration (DUR) for all regions showing a greater response to longer durations (red line), for regions that were sensitive to high SS (yellow line), for regions that were sensitive to low SS (blue line), and for regions showing no effects of SS or DUR (black line). Inset B: similar to Inset A, except that percent signal change is shown as a function of the SS variable. 
sure durations, although to varying degrees. Importantly, however, this interaction was not fully crossed for any of the dependent measures except bias, making the interpretation of this interaction somewhat more difficult. Consequently, we conducted simple main effects analyses (Keppel \& Zedeck, 1989) of SS at each exposure duration (for each dependent measure) to determine whether structural processing was evident at all exposure durations. For SENS and bias, the simple main effect of SS was significant at all three durations $(p<.05)$, whereas for ERR and RT, the simple main effect of SS was significant only for the two longer durations $(p<.05)$. On the basis of these analyses, we conclude that less structural processing occurred at the shortest duration and more structural processing occurred at longer durations. The shortest duration, however, made the task much more difficult, so the lack of structural processing in terms of RT and ERR may reflect a ceiling effect.

\section{Brain-Imaging Results}

Table 1 summarizes the results of the scalar correlations and repeated measures ANOVAs for ROIs showing significant effects, which are illustrated in Figure 4. A positive correlation between fMRI signal and SS or DUR indicates that fMRI signal is greater for high SS levels or longer durations, respectively. A positive correlation between fMRI signal and RT, ERR, or SENS reflects longer processing time, more errors, and greater sensitivity in discriminating same and different pairs, respectively. Finally, a positive correlation between fMRI signal and bias reflects a bias to respond different, whereas a negative correlation reflects a bias to respond same. Several cortical
A

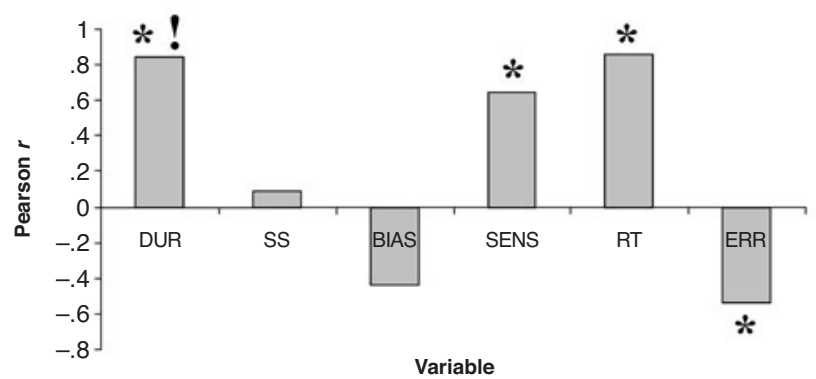

B

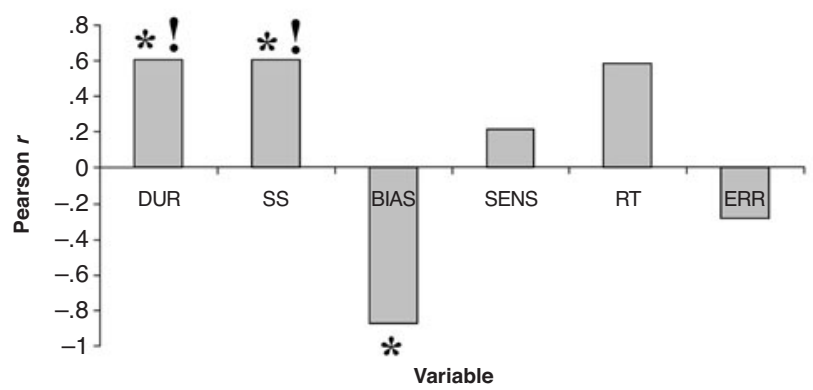

C

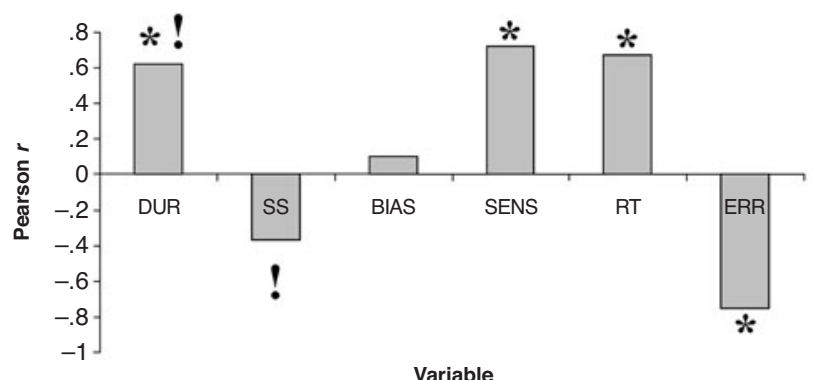

D

Right Cerebellum

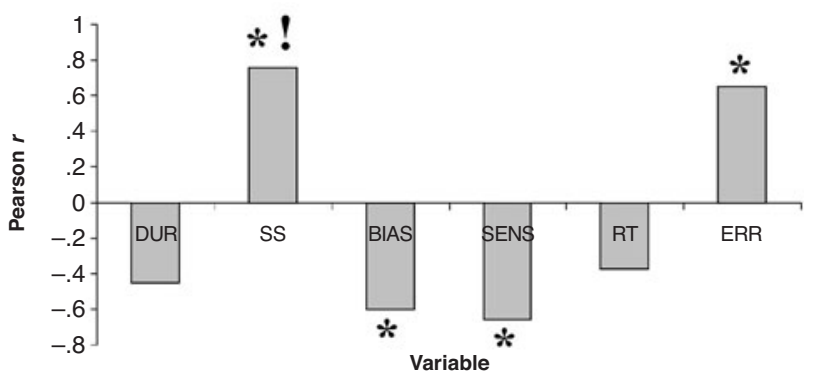

E

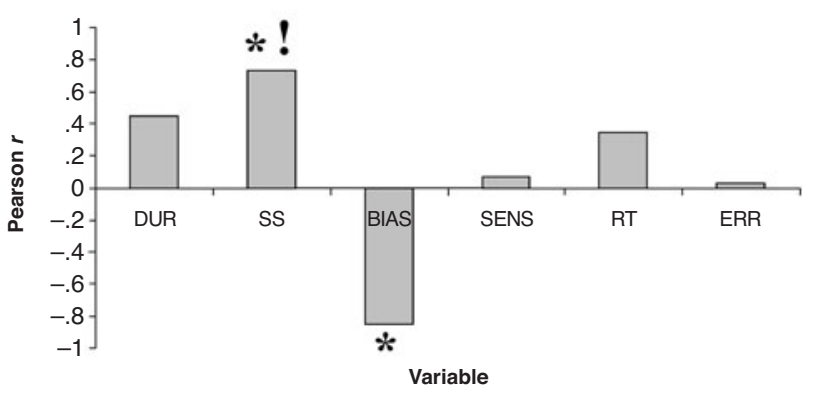

$\mathbf{F}$

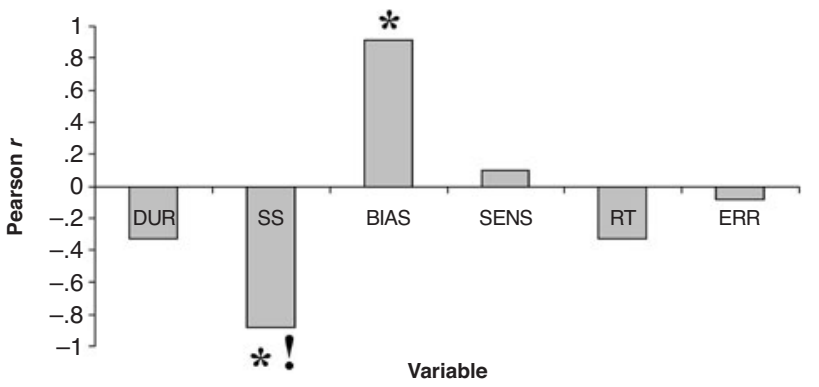

Figure 5. Correlation coefficients from Table 1 plotted for each independent variable (structural similarity [SS] and exposure duration [DUR]) and each performance measure (bias, sensitivity [SENS], reaction time [RT], and error rate [ERR]) in six cortical regions. The * indicates a significant Pearson $r$ and the ! indicates a significant main effect according to the ANOVAs. For panel A, the Pearson $r$ values reflect the average $r$ values across several different occipital and fusiform regions, and the ! and * indicate significant $F$ or $r$ values, respectively, in all individual regions (see Table 1). For panels B through F, the Pearson $r$ values reflect the value in the single region indicated. 
regions showed unique profiles as a function of these variables (Figure 5).

Occipital cortex and fusiform regions were particularly sensitive to the DUR manipulation (red voxels in Figure 4) and showed greater signal with longer exposure durations; however, these regions were not sensitive to the SS manipulation. With regard to performance measures, these regions showed significant correlations with RT, ERR and SENS, but none showed a correlation with bias (Figure 5A). The fMRI signal in these DURsensitive regions was associated with greater sensitivity in discriminating same and different picture pairs, longer RT, and fewer errors.

Two occipital and fusiform regions deviated from the response profile of much of the rest of the occipital and fusiform cortex. An anterior and ventral aspect of the left fusiform gyrus (lavFG, orange voxels in Figure 4) was sensitive to both DUR and SS, showing a greater signal for both longer durations and higher SS. In addition, this region reflected only one aspect of performance - namely, a bias to respond same (Figure 5B). An aspect of the right lingual gyrus showed a similar profile as the rest of the occipital cortex, with the exception of a significant main effect of SS according to the ANOVAs. The fMRI signal in the right lingual gyrus was greater with lower SS levels and with longer durations (Figure 5C).

Other brain regions were also sensitive to the SS manipulation. The fMRI signal in the left parietal cortex and the right cerebellum was greater for high-SS pairs than for low-SS pairs (yellow voxels in Figure 4) but was not affected by DUR. In the right cerebellum, the fMRI signal was associated with more errors, lower sensitivity and a bias to respond same but was not associated with processing speed (Figure 5D). In contrast, in the parietal cortex, the fMRI signal was associated with a bias to respond same but was not associated with RT, ERR, or SENS (Figure 5E). The right middle frontal gyrus (blue voxels in Figure 4) showed a greater response to low-SS than to high-SS pairs and was strongly associated with a bias to respond different (Figure 5F) but was not associated with other performance measures.

Inset A of Figure 4 shows the fMRI signal as a function of DUR in all regions that were modulated by DUR (red line) versus regions that were not. As was expected, the fMRI signal increased as DUR increased only in those regions that were modulated by DUR. Regions that were modulated by high or low SS or regions that showed no effects of either variable showed no effect of DUR. Inset B of the same figure shows the fMRI signal as a function of SS in all the regions that were modulated by SS (yellow line) versus the regions that were not. As was expected, the regions that were sensitive to high SS showed a linear increase in signal as SS increased (yellow or orange voxels), the regions that were sensitive to low SS (blue line) showed a linear decrease in signal as SS increased (blue or purple voxels), and the regions that were not modulated by SS showed no effect.

\section{DISCUSSION}

In the present study, we explored structural processing as a potential mechanism with which to explain the functional organization of the VPS. In addition to replicating our previous finding that greater demands on structural differentiation activated the anterior VPS (Joseph \& Gathers, 2003; Joseph et al., 2000), we also implicated many of the same cortical regions reported in other studies of object recognition, including occipital and posterior fusiform regions, the anterior fusiform gyrus, and the parietal and frontal cortex (Bar et al., 2001; Fize et al., 2000; Ishai, Ungerleider, Martin, \& Haxby, 2000; Koutstaal et al., 2001; Op de Beeck, Beatse, Wagemans, Sunaert, \& Van Hecke, 2000; Sugio et al., 1999). However, the novel contribution of the present study was showing that activation in these regions is associated with different aspects of object recognition performance. In the subsequent paragraphs, we will discuss the response profiles of the various brain regions involved in object recognition in the present study and will speculate on each region's contribution to the object recognition process.

\section{Occipital and Posterior Fusiform Regions}

Regions in the occipital and posterior fusiform cortex, as well as in some more anterior fusiform regions, were sensitive to the DUR manipulation, but not to the SS manipulation. These regions showed a greater signal for longer durations, similar to other findings in the literature (Grill-Spector, Kushnir, Hendler, \& Malach, 2000; Madden et al., 2002; Price \& Friston, 1997; Price, Moore, $\&$ Frackowiak, 1996; Price et al., 1994). Given that these regions were not sensitive to the SS manipulation, we conclude that they are not directly involved in processing object structure. Activation in these regions is driven by the amount of time a stimulus is displayed, rather than by similarity of object shape. With respect to performance measures, these regions also reflected longer processing time, decreased errors, and increased sensitivity. We suggest that the increased level of activation in occipital and fusiform regions with longer DUR provides more visual information to better perform the task; with more time to view a stimulus pair, subjects take more time to respond but perform better in terms of reduced error rates and increased sensitivity. In other words, activation in these regions reflected more successful task performance, which can be attributed to a longer viewing time of the object pairs. The present data cannot further define the type of processing that occurs here; we can conclude only that it is not related to the structural processing stage of object recognition, because the fMRI signal was not modulated by the SS manipulation.

\section{Left Anterior Ventral Fusiform Gyrus}

An lavFG region was sensitive to both SS and DUR manipulations, showing greater signal for longer durations and higher SS levels. This was the only region in 
the VPS to show sensitivity to the SS variable, and we have implicated similar regions as sensitive to high SS in other studies (Joseph \& Gathers, 2003; Joseph et al., 2000). Hence, our first prediction, that anterior fusiform regions would be sensitive to high SS, was upheld. We suggest that this region is specifically involved in structural processing during object recognition, given that it shows a greater signal to higher SS levels but does not show sensitivity to other types of visual similarity, such as color similarity (Joseph \& Steinmetz, 2003). With higher SS levels, perceptual information, such as global shape, is not sufficient to distinguish items in a pair, leading to the activation of structural representations. It is the processing of structural descriptions that we suggest occurs in relatively more anterior fusiform regions.

Both the present study and our previous one (Joseph \& Gathers, 2003) showed that the left anterior fusiform gyrus was more sensitive to structural similarity than was the right fusiform gyrus. In addition, the right fusiform gyrus was associated with greater sensitivity, longer RTs, and fewer errors, whereas the lavFG was sensitive only to response bias. Other neuroimaging studies of object and face recognition (Gauthier et al., 1999; Kanwisher et al., 1997) have implicated stronger responses in the right fusiform gyrus than in the left, implying that the right VPS may be more critical for visual recognition than the left. One explanation for this apparent discrepancy comes from a recent fMRI study of object recognition (Vuilleumier, Henson, Driver, \& Dolan, 2002) that concluded that the right fusiform gyrus is sensitive to viewpointdependent representations for objects, whereas the left fusiform gyrus is not sensitive to viewpoint-specific representations. Using visual field methodology, Burgund and Marsolek (2000) similarly concluded that the right hemisphere processes specific, viewpoint-dependent object representations, whereas the left hemisphere processes more abstract, viewpoint-invariant object descriptions. The present matching task may have made different demands on viewpoint-invariant object representations than did the tasks used in other studies. Specifically, in the present matching task, the two objects within a pair were frequently presented in different viewpoints, requiring matching across different viewpoints and, consequently, relying more on viewpoint-invariant representations. According to Vuillemier et al.'s conclusion, this reliance on viewpointinvariant representations may be subserved more by the left fusiform gyrus than by the right.

Another potential explanation for greater lefthemisphere involvement in structural object processing is that the left hemisphere is more often involved in analytic or feature-based processing, whereas the right hemisphere is more engaged during holistic processing across a wide range of different visual stimuli and tasks (Doricchi, Armati, Martorano, \& Violani, 1995; Moses et al., 2002; Parkin \& Williamson, 1987; Van Kleeck, 1989). In addition, detecting differences among stimuli is often considered a more analytic process, whereas detecting sameness or identity is often thought of as holistic (Miller, 1978). Lateralization studies have shown that different decisions are faster when stimuli are presented to the right visual field/left hemisphere and same decisions are faster when stimuli are presented to the left visual field/right hemisphere (Bradshaw, Gates, \& Patterson, 1976; Magnani, Mazzucchi, \& Parma, 1984). In the context of the present study, deciding that two structurally similar animals are different could make greater demands on the analysis of individual features or components of the objects. For two objects with very similar structural representations, such as camel and horse, most of the components are similar, and observers may rely on the detection of features, such as manes or humps, to differentiate the two objects. This kind of processing is inherently more analytic than holistic and would likely make greater demands on left-hemisphere structures.

With respect to our second objective, the present study extends previous findings by showing that one performance measure in particular - response bias-is strongly associated with activation in the lavFG, whereas other performance measures are not. We interpret this finding to indicate that activation in the lavFG reflects the accumulation of structural object information en route to recognition. Specifically, when two high-SS objects must be distinguished, demands on the processing of structural descriptions increases, leading to a greater signal in areas that process object structure-in this case, the lavFG. The goal of the observer is to determine whether the two objects are the same or different. However, with high-SS pairs, the activated structural descriptions are very similar to each other so the observer shifts the criterion setting to the same end of the response scale. In other words, the more similar two objects are in structure, the more likely it is that the observer will say that they are the same. This bias is driven by the similarity of the two objects at a structural level. However, a bias to respond same is not necessarily indicative of success or failure in object recognition; a liberal response bias will lead to more accurate performance when two objects are indeed the same but will lead to less accurate performance when two objects are different. Given that neither accuracy of responding nor SENS was correlated with fMRI signal in this region, we conclude that activation in the lavFG reflects the accumulation of structural information during object recognition but is not directly linked to success or failure in object recognition in this type of perceptual matching task. Importantly, SS-modulated activation in the lavFG cannot be attributed to greater generalized task difficulty. If this were the case, the fMRI signal in the lavFG region should have been correlated with high ERR and decreased SENS, which did not occur. Also, with respect to our third objective, if activation in the lavFG reflects generalized task difficulty, the fMRI signal in this region should have been correlated with shorter DUR (the most difficult of the DUR conditions), and it was not. Therefore, lavFG activation reflects structural processing, rather than generalized task difficulty.

If the lavFG region is indeed related to structural processing, one might expect the fMRI signal in this region to be correlated with SENS in addition to bias, given that 
SENS decreased as SS increased. However, in signal detection theory, SENS and bias are theoretically independent from each other (MacMillan \& Creelman, 1991). Two equally sensitive observers can adopt drastically different response biases. Therefore, it is conceivable that fMRI signal was correlated with bias, but not with SENS. In fact, behaviorally, SENS and bias were not correlated with each other, which also supports their independence. The fact that $\mathrm{fMRI}$ signal in this lavFG region was correlated with bias, but not with SENS, implies that the shape of the fMRI response function matched the shape of the bias function more closely than it matched the shape of the SENS function. In addition, although the SS manipulation affected both SENS and bias, SS influenced the bias measure more than it influenced the SENS measure (as is illustrated clearly in Figure 3). Consequently, if fMRI signal in a brain region was correlated with SS, it was also more likely to be correlated with bias than with SENS in this study. The fMRI signal in the lavFG reflects the most relevant aspect of behavior driven by SS - namely, changes in criterion settings that are related to different demands on structural processing.

Our conclusion that activation in the lavFG may not reflect successful object recognition performance appears to be at odds with other findings showing that successful object recognition is associated with brain activation levels in relatively anterior VPS areas (Bar et al., 2001; Grill-Spector et al., 2000; James et al., 2000). In those studies, the goal of the observer was to identify single objects either by retrieving the correct name silently (Grill-Spector et al., 2000; James et al., 2000) or by providing a subjective rating for correctness of response (Bar et al., 2001). If the fusiform gyrus is the site where information about object structure accumulates, this accumulation may be sufficient for successful singleobject recognition. However, in our perceptual matching task, the accumulation of structural object information in the fusiform gyrus is for two competing, but highly similar, structural representations. Therefore, the differences between the objects are not likely resolved at this site. Activation here reflects a bias to respond same because structural similarity is so high in the high-SS conditions but does not reflect accuracy of responding. We also note that the lavFG region is influenced by exposure duration. Behaviorally, longer durations enhanced the effect of high SS, and the signal in the lavFG reflects this.

We have suggested that changes in response bias as a function of SS reflect the accumulation of structural object information but do not reflect object recognition success or failure. Alternatively, the present block design may have encouraged the subjects to change criterion settings as a function of SS and DUR, so that changes in response bias are merely an artifact of blocking the major independent variables. Although our previously published study on this topic also blocked the SS variable (Joseph \& Gathers, 2003), other unpublished data in our lab show that when SS is not blocked, response bias changes in the same manner as in the present study. However, the mag- nitude of the change in response bias was greater in the present study than in our previous studies, even though the SS effect on bias was significant in both cases. Therefore, the effect of blocking may be to enhance the magnitude of bias values, but it does not appear to change the effect of SS leading to a more liberal response bias.

Although the fusiform gyrus is classically considered the major site for object recognition, the present findings indicate that this may not be the site where competing object representations are successfully disambiguated in a perceptual matching task. The final process of disambiguation may occur further downstream in regions involved in response selection or in controlling motor responses, or the final decision may involve feedback loops to other visual and nonvisual areas. These downstream regions and feedback sites may vary depending on whether the task involves naming, perceptual matching, episodic memory, and so forth. One complication in isolating a single region involved in the final decision process is that multiple brain regions may be activated in parallel. The lavFG activation in the present study may have occurred in parallel with occipital and frontal activation that was associated with successful recognition. Additional research is needed to determine the temporal order of activation and flow of information during object recognition.

\section{Right Lingual Gyrus}

The right lingual gyrus had a similar response profile as other occipital regions, with one exception: This region showed greater sensitivity to low SS than to high SS, according to the ANOVAs. We previously had found a similar dissociation between posterior and anterior VPS regions for both animal and 3-D shape matching, in which mid-to-anterior fusiform regions were sensitive to high SS and striate, extrastriate, and posterior fusiform regions were sensitive to low SS (Joseph \& Gathers, 2003). Activation in the right lingual gyrus in the present study does not reflect the structural processing stage of object recognition, given that this region was sensitive to low SS. Instead, this region reflects processing that is involved in discriminating objects that are very dissimilar in shape. One likely candidate for such a process is comparison of the global shapes of the two stimuli, which are sufficiently different from each other in the low-SS conditions. We therefore speculate that right lingual activation in the present study reflects a prestructural stage of processing most likely related to early visual processing such as computing global shape. However, a more direct test of this hypothesis is needed.

\section{Left Parietal Cortex}

The left parietal cortex showed sensitivity to high SS levels, but not to DUR, in the present study. Given this, we suggest that activation in left parietal regions is related to demands on processing structural descriptions in object recognition. Similar to the lavFG region that was also sensitive to high SS, the left parietal cortex reflected a bias to respond same but was not associated with other performance measures. Parietal activation has 
been reported in other functional brain-imaging studies of object recognition (Faillenot, Sunaert, Van Hecke, \& Orban, 2001; Ishai et al., 2000; James et al., 2000; Joseph \& Gathers, 2003; Koutstaal et al., 2001; Schendan \& Kutas, 2003; Sugio et al., 1999; Taira, Nose, Inoue, \& Tsutsui, 2001) and has often been interpreted as being related to mental rotation, image alignment or visual imagery processes. Conceivably, the parietal activation in the present study could reflect image alignment that is engaged when two objects are difficult to distinguish in terms of shape (high SS). In this case, further perceptual analysis of details or features of the objects may be more efficient if the two objects are aligned along the same axis. Importantly, though, parietal activation was not associated with ERRs, processing time, or SENS measures; hence, it reflects only a change in criterion settings as SS levels increase but does not necessarily reflect object recognition success or failure.

\section{Right Cerebellum}

The right midline cerebellum showed a greater response to high SS than to low SS and also reflected a bias to respond same, similar to that for the lavFG and the left parietal cortex. However, the fMRI signal in the right cerebellum also reflected poorer performance, in that it was associated with high error rates and decreased sensitivity between same and different pairs. Hence, it is dissociated from the other SS-sensitive regions in this regard. Because one of the major functional roles of the cerebellum is coordinating motor output, we speculate that the greater signal in the right cerebellum is related to execution of the incorrect response. This incorrect response occurs more frequently when SS is high, because highly similar but different object pairs are mistaken for same pairs.

\section{Right Frontal Cortex}

The right middle frontal gyrus (BA 6) showed a stronger response to low-SS pairs than to high-SS pairs and also reflected a bias to respond different. Clearly, this response profile reflects processing when two objects are very dissimilar in shape. Because activation in this region was not associated with any other performance measures, it was not directly involved in object recognition success or failure but was instrumental in shifting the criterion setting to a more conservative value. Frontal activation has been reported in a number of neuroimaging studies of object recognition, and some suggested functional contributions by frontal sites include discriminating target from nontarget objects in object categorization (VanRullen $\&$ Thorpe, 2001), selecting an object representation from multiple candidates (Schendan \& Kutas, 2003), or providing a best guess as to an object representation in order to constrain further perceptual or structural analysis (Bar, 2003). Although these proposals may reflect different aspects of the same process, the latter suggestion seems most compatible with the present data, as will be discussed further below.
Recently, Bar (2003) has suggested a processing account for top-down facilitation in object recognition, which is relevant to the present findings. Specifically, he proposes that low spatial frequency information in an image is processed in early visual regions, then rapidly projected to the prefrontal cortex as a means to make an initial best guess as to an object's identity. Information from prefrontal regions is then projected to ventral temporal regions, where additional processing occursincluding high spatial frequency analysis. Although the present study cannot address the temporal order or duration of different components of the object recognition process, the present data seem compatible with Bar's account. When two objects of a pair are low in SS, low spatial frequency information (e.g., global outlines or orientations) may be sufficient to differentiate the two objects. In Bar's account, this would result in activation in early visual areas and prefrontal regions. The prefrontal activation would reflect the generation of two distinct sets of candidate object representations - one set for each object in the pair. Indeed, in the present study, both the right lingual gyrus and the right middle frontal gyrus were more responsive to low SS than to high SS (albeit our right frontal region is different from those that Bar proposed as candidates for low spatial frequency processing). In the case of high-SS pairs, however, the low spatial frequency information is not sufficient for deciding that the two objects are different. Consequently, prefrontal cortex activation is associated with generating either two highly overlapping candidate representations or a single candidate representation, given the high similarity of the objects in high-SS pairs. In high-SS conditions, then, the left anterior fusiform gyrus is activated, which may represent the additional processing required when low spatial frequency information does not suffice for making the decision. Such a processing account provides an intriguing mechanistic approach to object recognition but will need to be tested in additional studies to further characterize the neural substrates of structural differentiation in object recognition.

\section{CONCLUSION}

The present study showed that anterior fusiform regions are sensitive to greater demands on the structural processing stage of object recognition, whereas other fusiform and early visual areas are not, which replicates our previous findings (Joseph \& Gathers, 2003; Joseph et al., 2000). In addition, the fMRI signal in the frontal and parietal cortex and the cerebellum was sensitive to demands on structural object processing, but visual areas (the primary visual, extrastriate, and fusiform cortex) were sensitive only to the DUR manipulation. Consequently, demands on object processing, as indexed by the manipulation of structural similarity, recruit a network of brain regions, whereas varying the DUR of a visual image affects only visual regions. Most important, the novel finding was that different components of the ob- 
ject recognition network are affiliated with different aspects of task performance. The brain-imaging data alone describe the regions involved in object recognition, but these data do not tease apart the different components of the object recognition process, which is the goal of cognitive neuroscience. Although it may be no surprise that the lavFG is an important contributor to this object recognition task, the neural processing in this region is, surprisingly, not associated with successful object recognition performance. Instead, other occipital and fusiform regions contribute to successful object recognition in this particular task. Therefore, the performance measures tell us that different aspects of the VPS are linked to different components of the object recognition process. Moreover, future studies may reveal that the cortical regions that are associated with the same aspects of behavior are more strongly functionally connected than are the regions that are not linked to the same performance measures.

\section{REFERENCES}

BAR, M. (2003). A cortical mechanism for triggering top-down facilitation in visual object recognition. Journal of Cognitive Neuroscience, 15, 600-609.

Bar, M., Tootell, R. B. H., Schacter, D. L., Greve, D. N., FisChl, B., Mendola, J. D., Rosen, B. R., \& Dale, A. M. (2001). Cortical mechanisms specific to explicit object recognition. Neuron, 29 , 529-535.

BAUER, R. M. (1993). Agnosia. In K. M. Heilman \& E. Valenstein (Eds.), Clinical neuropsychology (3rd ed., pp. 215-277). New York: Oxford University Press.

Biederman, I. (1987). Recognition-by-components: A theory of human image understanding. Psychological Review, 94, 115-147.

Bradshaw, J. L., Gates, A., \& Patterson, K. (1976). Hemispheric differences in processing visual patterns. Quarterly Journal of Experimental Psychology, 28, 667-681.

BRUCE, V., \& HUMPHREYS, G. W. (1994). Recognizing objects and faces. Visual Cognition, 1, 141-180.

BURGUND, E. D., \& MARSOLEK, C. J. (2000). Viewpoint-invariant and viewpoint-dependent object recognition in dissociable neural subsystems. Psychonomic Bulletin \& Review, 7, 480-489.

Damasio, A. R., Damasio, H., \& Van Hoesen, G. W. (1982). Prosopagnosia: Anatomic basis and behavioral mechanisms. Neurology, $\mathbf{3 2}$ 331-341.

Damasio, H., Grabowski, T. J., Tranel, D., Hichwa, R. D., \& DamaSIO, A. R. (1996). A neural basis for lexical retrieval. Nature, 380 , 499-505.

DONALDSON, W. (1992). Measuring recognition memory. Journal of Experimental Psychology: General, 121, 275-277.

Doricchi, F., Armati, C., Martorano, G., \& Violani, C. (1995). Generation of skeletal and multipart mental visual images in the cerebral hemispheres: A study in normal subjects. Neuropsychologia, $\mathbf{3 3}$, 181-201.

Downing, P. E., Jiang, Y., Shulman, M., \& Kanwisher, N. (2001). A cortical area selective for visual processing of the human body. Science, 293, 2470-2473.

EPSTEIN, R., \& KANWISHER, N. (1998). A cortical representation of the local visual environment. Nature, 392, 598-601.

Faillenot, I., Sunaert, S., VAn Hecke, P., \& Orban, G. A. (2001). Orientation discrimination of objects and gratings compared: An fMRI study. European Journal of Neuroscience, 13, 585-596.

FARAH, M. J. (1990). Visual agnosia: Disorders of object recognition and what they tell us about normal vision (4th ed.). Cambridge, MA: MIT Press.

Fize, D., Boulanouar, K., Chatel, Y., Ranjeva, J. P., FabreThorpe, M., \& Thorpe, S. (2000). Brain areas involved in rapid categorization of natural images: An event-related fMRI study. NeuroImage, 11, 634-643.
Friston, K. J., Worsley, K. J., Frackowiak, R. S. J., Mazziotta, J. C., \& Evans, A. C. (1994). Assessing the significance of focal activations using their spatial extent. Human Brain Mapping, 1, 210-220.

Garrett, A. S., Flowers, D. L., AbSher, J. R., FAHEY, F. H., GaGe, H. D., Keyes, J. W., Porrino, L. J., \& Wood, F. B. (2000). Cortical activity related to accuracy of letter recognition. NeuroImage, 11, 111-123.

Gauthier, I., Tarr, M. J., Anderson, A. W., SkUdlarski, P., \& Gore, J. C. (1999). Activation of the middle fusiform " "face area" increases with expertise in recognizing novel objects. Nature Neuroscience, 2, 568-573.

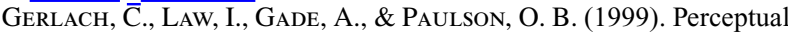
differentiation and category effects in normal object recognition. Brain, 122, 2159-2170.

GRILl-SPECTOR, K., KOURTZI, Z., \& KANwisher, N. (2001). The lateral occipital complex and its role in object recognition. Vision Research, 41, 1409-1422.

GRILL-SPeCtor, K., Kushnir, T., Hendler, T., \& Malach, R. (2000). The dynamics of object-selective activation correlate with recognition performance in humans. Nature Neuroscience, 3, 837-843.

Gur, R. C., Ragland, D., Harper Mozley, L., Mozley, P. D., Smith, R., Alavi, A., Bilker, W., \& Gur, R. E. (1997). Lateralized changes in regional cerebral blood flow during performance of verbal and facial recognition tasks: Correlations with performance and "effort." Brain \& Cognition, 33, 388-414.

Haxby, J. V., Ishai, A., Chao, L. L., Ungerleider, L. G., \& MarTIN, A. (2000). Object-form topology in the ventral temporal lobe. Trends in Cognitive Sciences, 4, 3-4.

HiLlis, A. E., \& CARAMAZZA, A. (1991). Category-specific naming and comprehension impairment: A double dissociation. Brain, 114, 2081-2094.

HUMPhREYS, G. W., RidDOCH, M. J., \& Quinlan, P. T. (1988). Cascade processes in picture identification. Cognitive Neuropsychology, $\mathbf{5}$, 67-103.

Ishai, A., Ungerleider, L. G., Martin, A., \& Haxby, J. V. (2000). The representation of objects in the human occipital and temporal cortex. Journal of Cognitive Neuroscience, 12, (Suppl. 2), 35-51.

James, T. W., Humphrey, G. K., Gati, J. S., Menon, R. S., \& Goodale, M. A. (2000). The effects of visual object priming on brain activation before and after recognition. Current Biology, 10, 10171024.

JoSEPH, J. E. (1997). Color processing in object verification. Acta Psychologica, 97, 95-127.

JOSEPH, J. E., \& GATHERS, A. D. (2002). Natural and manufactured objects activate the "fusiform face area." NeuroReport, 13, 935-938.

JosePh, J. E., \& GATHERS, A. D. (2003). Effects of structural similarity on neural substrates for object recognition. Cognitive, Affective, \& Behavioral Neuroscience, 3, 1-16.

JoSEPH, J. E., Jones, K. M., ZEFFIRO, T. A., \& EDEN, G. F. (2000). FMRI correlates of structural similarity in object naming. Society for Neuroscience Abstracts, 26 (Pt. 2), 1502.

JosePh, J. E., \& ProfFitT, D. R. (1996). Semantic versus perceptual influences of color in object recognition. Journal of Experimental Psychology: Learning, Memory, \& Cognition, 22, 407-429.

JoSEPH, J. E., \& STEINMETZ, N. A. (2003, June). Resolving shape and color similarity in the human ventral processing stream. Poster presented at the 9th International Conference on Functional Mapping of the Human Brain, New York.

Kanwisher, N., McDermott, J., \& Chun, M. M. (1997). The fusiform face area: A module in human extrastriate cortex specialized for face perception. Journal of Neuroscience, 17, 4301-4311.

KePpel, G., \& Zedeck, S. (1989). Data analysis for research designs. New York: Freeman.

Koutstaal, W., Wagner, A. D., Rotte, M., Maril, A., Buckner, R. L., \& SCHACTER, D. L. (2001). Perceptual specificity in visual object priming: Functional magnetic resonance imaging evidence for a laterality difference in fusiform cortex. Neuropsychologia, 39, 184-199.

Lazar, N. A., Luna, B., Sweeney, J. A., \& EDDY, W. F. (2002). Combining brains: A survey of methods for statistical pooling of information. NeuroImage, 16, 538-550.

Loftus, G. R., \& MASSON, M. E. J. (1994). Using confidence intervals in within-subjects designs. Psychonomic Bulletin \& Review, 1, 476490. 
MacMillan, N. A., \& Creelman, C. D. (1991). Detection theory: A user's guide. New York: Cambridge University Press.

Madden, D. J., Langley, L. K., Denny, L. L., Turkington, T. G., Provenzale, J. M., Hawk, T. C., \& Coleman, R. E. (2002). Adult age differences in visual word identification: Functional neuroanatomy by positron emission tomography. Brain \& Cognition, 49, 297-321.

Magnani, G., Mazzucchi, A., \& PARMa, M. (1984). Interhemispheric differences in same versus different judgments upon presentation of complex visual stimuli. Neuropsychologia, 22, 527-530.

Malach, R., Reppas, J. B., Benson, R. R., KWONG, K. K., Jiang, H., Kennedy, W. A., Ledden, P. J., Brady, T. J., Rosen, B. R., \& Tootell, R. B. H. (1995). Object-related activity revealed by functional magnetic resonance imaging in human occipital cortex. Proceedings of the $\mathrm{Na}$ tional Academy of Sciences, 92, 8135-8139.

MARR, D., \& NishIHARA, H. K. (1978). Representation and recognition of the spatial organization of three-dimensional shapes. Proceedings of the Royal Society of London: Series B, 200, 269-294.

MARTIN, A., \& CARAmazZA, A. (2003). Neuropsychological and neuroimaging perspectives on conceptual knowledge: An introduction. Cognitive Neuropsychology, 20, 195-212.

Martin, A., Wiggs, C. L., UNGERLEIDER, L., \& Haxby, J. (1996). Neural correlates of category-specific knowledge. Nature, 379, 649652.

Miller, J. (1978). Multidimensional same-different judgments: Evidence against independent comparisons of dimensions. Journal of Experimental Psychology: Human Perception \& Performance, 4 411-422.

Moses, P., Roe, K., Buxton, R., Wong, E., Frank, L. R., \& Stiles, J. (2002). Functional MRI of global and local processing in children. NeuroImage, 16, 415-424.

O'Brien, R., \& KAISER, M. (1985). MANOVA method for analyzing repeated measures designs: An extensive primer. Psychological Bulletin, 97, 316-333.

OKADA, T., TanaKa, S., Nakai, T., Nishizawa, S., InUi, T., SADATO, N.,YoneKura, Y., \& Konishi, J. (2000). Naming of animals and tools: A functional magnetic resonance imaging study of categorical differences in the human brain areas commonly used for naming visually presented objects. Neuroscience Letters, 296, 33-36.

Op de Beeck, H., Beatse, E., Wagemans, J., Sunaert, S., \& Van HeCKE, P. (2000). The representation of shape in the context of visual object categorization tasks. NeuroImage, 12, 28-40.

Papoulis, A. (1965). Probability, random variables, and stochastic processes. New York: McGraw-Hill.

Parkin, A., \& Williamson, P. (1987). Cerebral lateralization at different stages of facial processing. Cortex, 23, 99-110.

Price, C. J., \& Friston, K. J. (1997). The temporal dynamics of reading: A PET study. Proceedings of the Royal Society of London: Series $B, \mathbf{2 6 4}, 1785-1791$.

Price, C. J., Moore, C. J., \& Frackowiak, R. S. (1996). The effect of varying stimulus rate and duration on brain activity during reading. NeuroImage, 3, 40-52.

Price, C. J., Noppeney, U., Phillips, J., \& Devlin, J. T. (2003). How is the fusiform gyrus related to category-specificity? Cognitive Neuropsychology, 20, 561-574.

Price, C. J., Wise, R. J., Watson, J. D., Patterson, K., Howard, D., \& FrackowIAK, R. S. (1994). Brain activity during reading: The effects of exposure duration and task. Brain, 117, 1255-1269.

Schacter, D. L., Reiman, E., Uecker, A., Polster, M. R., Yun, L. S., \& CoOper, L. A. (1995). Brain regions associated with retrieval of structurally coherent visual information. Nature, 376, 587-590.

Schendan, H. E., \& Kutas, M. (2003). Time course of processes and representations supporting visual object identification and memory. Journal of Cognitive Neuroscience, 15, 111-135.

Spitzer, M., KischKa, U., GucKel, F., BellemanN, M. E., Kammer, T., Seyyedi, S., Weisbrod, M., Schwartz, A., \& Brix, G. (1998). Functional magnetic resonance imaging of category-specific cortical activation: Evidence for semantic maps. Cognitive Brain Research, 6, 309-319.

Sugio, T., Inui, T., Matsuo, K., Matsuzawa, M., Glover, G. H., \& NAKAI, T. (1999). The role of the posterior parietal cortex in human object recognition: A functional magnetic resonance imaging study. Neuroscience Letters, 276, 45-48.
TAira, M., Nose, I., Inoue, K., \& Tsutsui, K. (2001). Cortical areas related to attention to 3D surface structures based on shading: An fMRI study. NeuroImage, 14, 959-966.

TAlairaCH, J., \& TOURNOUX, P. (1988). Co-planar stereotaxic atlas of the human brain (M. Rayport, Trans.). New York: Thieme Medical.

TANAKa, K. (1996). Inferotemporal cortex and object vision. Annual Review of Neuroscience, 19, 109-139.

Tranel, D., Logan, C., FranK, R. J., \& Damasio, A. R. (1997). Explaining category-related effects in the retrieval of conceptual and lexical knowledge for concrete entities: Operationalization and analysis of factors. Neuropsychologia, 32, 1329-1339.

Tyler, L. K., Bright, P., Dick, E., Tavares, P., Pilgrim, L., Fletcher, P., Greer, M., \& Moss, H. E. (2003). Do semantic categories activate distinct cortical regions? Evidence for a distributed neural semantic system. Cognitive Neuropsychology, 20, 541-559.

Tyler, L. K., \& Moss, H. E. (2001). Toward a distributed account of conceptual knowledge. Trends in Cognitive Sciences, 5, 244-252.

Tyler, L. K., Stamatkis, E. A., Bright, P., Acres, K., AbDallah, S., RodD, J. M., \& Moss, H. E. (2004). Processing objects at different levels of specificity. Journal of Cognitive Neuroscience, 16, 351-362.

Ungerleider, L. G., \& MishKIN, M. (1982). Two cortical visual systems. In D. J. Ingle, M. A. Goodale, \& R. J. W. Mansfield (Eds.), Analysis of visual behavior (pp. 549-586). Cambridge, MA: MIT Press.

VAN KLEeCK, M. H. (1989). Hemispheric differences in global versus local processing of hierarchical visual stimuli by normal subjects: New data and a meta-analysis of previous studies. Neuropsychologia, 27, 1165-1178.

VANRULlen, R., \& THORPE, S. J. (2001). The time course of visual processing: From early perception to decision-making. Journal of Cognitive Neuroscience, 13, 454-461.

Vuilleumier, P., Henson, R. N., Driver, J., \& Dolan, R. J. (2002). Multiple levels of visual object constancy revealed by event-related fMRI of repetition priming. Nature Neuroscience, 5, 491-499.

Warrington, E., \& Shallice, T. (1984). Category specific semantic impairments. Brain, 107, 829-854.

Woods, R. P., CHERry, S. R., \& MAZziotta, J. C. (1992). Rapid automated algorithm for aligning and reslicing PET images. Journal of Computer Assisted Tomography, 16, 620-633.

\section{NOTES}

1. Although high- and low-SS naming activated dissociated regions in much of the VPS, both high- and low-SS naming activated different aspects of the temporal pole. Consequently, the anterior-posterior distribution of activation applied primarily to the fusiform and inferior temporal gyri.

2. The complement for the DUR variable was the difference between 2,000 and each DUR value, because 2,000 msec was the maximum amount of time per trial; the complement for the SS variable was the difference between 1,000 and each SS value, because 1,000 was the maximum possible rating in the previous study that generated the similarity ratings (Joseph, 1997).

3. We also conducted a random-effects analysis that yielded similar but not identical results. In general, the clusters of activation in that analysis were not as extensive or dense as those listed in Table 1.

4. In the ROI analysis, the reference waveforms were those depicted in Figure 1; that is, the FIX condition was assigned the value of 0 for the cross-correlations in order to maximize detecting activation greater than the fixation condition. Note that the SS and SS-complement waveforms, however, are not truly inverses of each other, due to this constraint. However, when we performed the scalar correlations within ROIs, we expressed fMRI signal as percent change relative to fixation, thereby dropping the FIX data point from the time series. By dropping the FIX data point, the SS and SS-complement reference waveforms are now truly inverses of each other.

(Manuscript received February 4, 2004; revision accepted for publication June 28, 2004.) 